\title{
La matrone, la louve et le soldat : pourquoi des prostitué(e)s « ingénues » à Rome?
}

Florence DUPONT

\section{(2) OpenEdition \\ 1 Journals}

Édition électronique

URL : https://journals.openedition.org/clio/580

DOI : $10.4000 /$ clio. 580

ISSN : 1777-5299

Éditeur

Belin

Édition imprimée

Date de publication : 1 avril 2003

Pagination : 21-44

ISBN : 2-85816-663-3

ISSN : $1252-7017$

Référence électronique

Florence DUPONT, «La matrone, la louve et le soldat : pourquoi des prostitué(e)s « ingénues » à Rome? ", Clio. Histoire, femmes et sociétés [En ligne], 17 | 2003, mis en ligne le 14 novembre 2006, consulté le 22 avril 2022. URL : http://journals.openedition.org/clio/580 ; DOI : https://doi.org/ 10.4000/clio.580

Ce document a été généré automatiquement le 22 avril 2022.

Tous droits réservés 


\title{
La matrone, la louve et le soldat: pourquoi des prostitué(e)s « ingénues » à Rome?
}

\author{
Florence DUPONT
}

Le don sexuel et le marché des corps : l'économie de la prostitution romaine

1 La prostitution à Rome peut sembler un sujet bien connu et suffisamment étudié ${ }^{1}$ : que l'on pense aux lupanars de Pompéi et leurs fresques "pornographiques ", ou à ces créatures aussi vénales qu'affolantes que sont les courtisanes de comédie, ou encore à ces figures de "demi-mondaines $»^{2}$ que célèbreraient les poètes, Properce, Horace ou Catulle, sans parler des princesses impériales ${ }^{3}$, comme Julie ou Messaline, dont les historiens anciens dénoncent à plaisir les incartades sexuelles la nuit dans Rome ${ }^{4}$. Enfin les hommes eux-mêmes sont accusés de se prostituer comme César et son ami Mamurra dont Catulle prétend qu'ils disputaient leurs clients aux filles des rues. Toutes ces figures peuplent la Rome imaginaire de nos contemporains, dont ils font volontiers une société orgiaque et "décadente». Or cet imaginaire doit être soumis à une rigoureuse révision historique car dans ces quelques exemples, à l'évidence, le mot de prostitution renvoie tantôt au commerce réel des corps tantôt à des pratiques sexuelles féminines, ou masculines, marginales que ce terme sert métaphoriquement à stigmatiser.

Définition économique

2 Il faut, en effet, distinguer la pratique réelle de la prostitution c'est-à-dire le commerce monnayé des corps, d'avec la diatribe moralisatrice qui accuse de prostitution quiconque à Rome, en dehors des prostitué(e)s reconnu(e)s et déclaré(e)s, s'adonne à une sexualité transgressive par rapport aux normes de la société romaine. Bien souvent les textes latins usent de la métaphore de la prostitution pour attaquer l'une ou l'autre, l'un ou l'autre. Il convient donc de trier sévèrement les documents. Sinon l'on regardera comme prostitué(e)s précisément ceux et celles qui ne le sont pas dans la mesure où l'accusation morale de prostitution peut être utilisée contre eux. Clodia, par exemple, que Catulle invective sous le nom de Lesbie ou encore que Cicéron attaque grossièrement dans le Pro Caelio et qu'il traite de prostituée à quatre sous, n'a jamais été 
une meretrix au sens propre ${ }^{5}$. Fille, sœur, épouse puis veuve de patricien, elle n'a jamais perdu ce statut honorable de matrone. Ou encore Poppée concubine de Néron ${ }^{6}$ et que Tacite qualifie métaphoriquement de "putain"-scortum. Il en est de même de Cléopâtre traitée de « reine prostituée » - regina meretrix ${ }^{7}$.

3 Certes quand un texte latin utilise le terme de prostituée pour figurer l'immoralité d'une femme ou d'un homme, cet usage métaphorique apporte des informations à l'historien sur le statut infamant de ce groupe social et donc peut et même doit être pris en compte mais de façon médiate, car avant d'être une figure de l'immoralité, la prostitution est une pratique sociale reconnue par la cité romaine et devant être étudiée comme telle.

Outre cette distinction à faire entre réalité et métaphore, ce que nous appelons de nos jours la prostitution recouvre plusieurs pratiques romaines distinctes dont certaines ne sont pas considérées comme telles par les Romains. En effet, on ne peut pas assimiler à la prostitution la location d'une esclave par son maître à un étranger, pour un temps plus ou moins long, et les prestations sexuelles qu'elle doit à ce maître parce qu'elle est sa propriété. De la même façon une affranchie peut vendre ses prestations sexuelles mais se doit gratuitement à son patron bien que sur un mode différent de celui de l'esclave. Il convient donc de restreindre la notion à une prostitution strictement définie en termes d'échanges marchands : prestations sexuelles contre argent.

5 En fait aucune étude historique sérieuse, à notre connaissance, n'a été faite jusqu'à présent qui prenne en compte la signification culturelle de cette activité, en la replaçant dans l'économie romaine des corps, c'est-à-dire un système social hiérarchisé où interfèrent des échanges marchands et des pratiques de don ${ }^{8}$. Ce qui donne lieu à des combinaisons multiples dont certaines relèvent de la prostitution et d'autres non.

Les rapports économiques à Rome, en effet, comme dans bien des sociétés traditionnelles, peuvent relever soit du don et du contre-don, soit de l'échange marchand. Dans le premier cas les services sexuels sont inclus à Rome dans des relations de clientèle, il s'agit d'un officium, d'un "devoir", de l'affranchi(e) à son ancien maître, dans l'autre il s'agit d'une véritable prostitution, d'un commerce monnayé. À cela s'ajoute une autre situation économique : celle de l'esclave qui est une marchandise - mancipium - et dont le corps est à la disposition de son maître.

7 En dehors des échanges marchands - et en dehors du mariage - voici comment se répartissent les prestations sexuelles possibles et impossibles entre un Romain libre et ses partenaires potentiels : "La soumission sexuelle - impudicitia -, dans le cas d'un homme libre peut être matière à procès, c'est une contrainte de la servitude, c'est un service que l'affranchi doit à son patron $»^{9}$.

8 Formulée dans le cas d'un partenaire masculin, la règle serait la même pour une partenaire féminine. Tout(e) affranchi(e) est donc a priori susceptible de complaisances sexuelles à l'égard de son patronus. Il en est de même des esclaves. Esclaves et affanchi(e)s sont impudici et par conséquent infâmes par leur statut. En revanche le don sexuel est impossible entre personnes libres, nées libres.

9 Si donc on définit la prostitution comme l'échange marchand de prestations sexuelles, échappent normalement à cette définition d'une part les prestations sexuelles imposées aux esclaves puisqu'ils sont la propriété de leur maître, d'autre part celles qui sont insérées dans les relations de don et de contre-don exigées par le clientélisme romain. Or c'est dans cet espace du don que se réalisent les prestations sexuelles «libres », en- 
dehors de la sexualité conjugale, où des hommes libres ont pour partenaires des affranchi(e)s. Il faut y ajouter la sexualité domestique entre les maîtres - pères et fils de famille - et la population servile de la maison, masculine et féminine dès l'âge le plus tendre.

Maintenant la prostitution ainsi définie et limitée aux seuls échanges marchands des corps, peut être le fait soit d'esclaves, loués par leur maître, soit d'affranchi(e)s soit d'hommes et de femmes libres, nés libres, ceux que les Romains appellent - ingenui - des ingénu(e)s qui vendent leurs prestations sexuelles. En fait les seul(e)s prostitué(e)s dont le droit et la morale se soucient sont les ingénus, faisant commerce de leurs corps de façon ouverte et notable. En effet, d'une façon générale l'impudicitia volontaire et en particulier la prostitution, ne peut déshonorer qu'un homme ou une femme susceptibles d'être honorables, ce que ne sont pas les affranchi(e)s. L'homme libre, volontairement impudicus risque d'être officiellement marqué d'infamie par le censeur, ce qui le privera de certains droits, si au contraire, il a été soumis par la contrainte, c'est un

crime - stuprum - commis par le violeur contre l'honneur - pudor - d'un corps libre, né libre. Il en est de même des femmes. La prostitution est donc une alternative au stuprum pour un corps libre et pour aucun autre, car en droit la prostitution rend impossible le stuprum. Les termes latins du droit sont explicites : à l'égard de celui ou celle qui fait commerce de son corps - corpora quaestoria - il n'y a pas de crime sexuel possible - in quo stuprum non committitur ${ }^{10}$. Autrement dit il n'y a pas de poursuite possible contre le violeur ou le séducteur, à l'égard de la femme libre, née libre, mariée ou non qui fait commerce de son corps. Sinon il y a stuprum. Il en est de même des hommes. Tout cela peut se résumer dans le tableau suivant:

\begin{tabular}{|l|l|l|l|}
\hline & Ingénu(e) & Affranchi(e) & Esclave \\
\hline \hline Don sexuel & impossible & Au patron & impossible \\
\hline \hline Commerce sexuel & Se vend & Se vend & Vendu(e) par son maître \\
\hline \hline Contrainte sexuelle & impossible & impossible & Objet sexuel \\
\hline
\end{tabular}

11 Finalement la prostitution au sens strict est à Rome quantitativement moins importante que dans nos sociétés égalitaires et démocratiques. Et elle n'est problématique que pour les prostitué(e)s ingénu(e)s. Il convient même de se demander pourquoi la prostitution existe puisque les esclaves et les affranchis pourraient sembler devoir suffire dans le cadre domestique à la satisfaction sexuelle des hommes libres en dehors des relations du type matrimonial.

Pourquoi des prostitué(e)s à Rome?

12 La réponse à cette question tient à la place et la nature des loisirs - otium -voluptueux dans la vie romaine et au coût des plaisirs qu'ils imposent. C'est pourquoi penser la prostitution romaine doit se faire d'abord en termes de dépenses somptuaires ${ }^{11}$. D'ailleurs les Romains ne censurent jamais l'usage des prostitué(e)s proprement dit(e)s pour des raisons morales mais allèguent toujours de raisons économiques : payer des prostitué(e)s menace les patrimoines comme toutes les autres dépenses en argent 
destinées aux plaisirs de l'otium que ce soit l'achat au marché de nourritures coûteuses - viandes et surtout poissons - et de parfums orientaux ou la location d'un cuisinier.

Malgré le blâme moral adressé aux prodigues excessifs, les dépenses destinées aux plaisirs de l'otium et en particulier l'argent payé aux prostitué(e)s sont à Rome une nécessité culturelle. En effet il est convenu au sein de la culture romaine que les jeunes hommes non mariés peuvent et doivent s'adonner aux divers plaisirs associés au banquet: le vin, la bonne chère et les filles (ou les garçons). La culture du plaisir est constitutive de l'otium c'est-à-dire de la structure générale de la vie du Romain ${ }^{12}$. Mais ces plaisirs juvéniles n'ont pas lieu, en général, dans la maison du père mais dans les maisons de prostitution à cause de leur caractère exceptionnel et excessif. D'une façon générale ces fêtes excèdent les possibilités autarciques d'une maison, aussi bien en ce qui concerne les nourritures qui sont achetées au marché que le personnel qui est loué pour l'occasion, cuisinier et prostitué(e)s qui en outre dansent, chantent et jouent de la musique. Un ou une esclave assez joli(e) pour n'être qu'un objet de plaisirs coûte trop cher pour être présent(e) dans de nombreuses maisons. Les multiplier mène à la ruine. C'est pourquoi la cité a besoin de prostitué(e)s pour ses plaisirs.

En outre les voyageurs et les soldats qui eux aussi s'adonnent aux plaisirs de l'otium à la fin du voyage ou lors des longues attentes hivernales, utilisent aussi les services des prostitué(e)s pour leurs banquets. Ainsi à côté de la population servile toujours sexuellement disponible, les Romains font appel à des prostitué(e)s afin de donner une dimension somptuaire, luxueuse, indispensable aux plaisirs raffinés qui constitue ce pôle indispensable de la vie civilisée qu'est l'otium urbanum, les loisirs de la ville. Ces plaisirs sont énumérés ainsi par Cicéron pour un noble romain: "les plaisirs du banquet, des jeux et des prostitué(e)s - scortorum $-»^{13}$ et se résument de la façon suivante pour un simple paysan en goguette: "ne rien faire, se promener, aller au cirque, au théâtre jouer aux dés, fréquenter les tavernes et les bordels ${ }^{14}$.

Les prostitué(e)s appartiennent donc à la population urbaine et sont des composantes indispensables à la civilisation telle que la conçoivent les Romains, au même titre que le vin, la musique, la cuisine, les parfums et tous les autres plaisirs du banquet. La prostitution est une pratique non pas sexuellement mais culturellement nécessaire à Rome.

Pourquoi des prostitué(e)s libres et ingénu(e)s?

16 Même réduite à des échanges marchands, comme on l'a vu dans le schéma général du commerce sexuel, il n'est pas possible à Rome de parler de prostitution au singulier puisque la société romaine connaît différents types de corps sexuellement soumis, renvoyant à des statuts sociaux différents : les esclaves, les affranchi(e)s, les hommes ou femmes libres. En outre même si les hommes se prostituent comme les femmes, les effets sociaux ne sont pas les mêmes pour les uns et les autres.

17 Face à ces diverses formes de prostitutions, notre travail se limitera à une population précise : celle des prostitué(e)s libres, né(e)s libres qui font commerce de leurs corps, celle qui pose le problème historique le plus intriguant. En effet on peut se demander : pourquoi existaient-ils des prostitué(e)s libres, ou plus précisément ingénu(e)s, libres et né(e)s de parents libres? Si la prostitution est nécessaire à l'otium urbanum, on ne comprend pas a priori pourquoi ce ne sont pas seulement des esclaves ou à la rigueur des affranchi(e)s qui sont utilisé(e)s dans les maisons de prostitution ou qui se vendent selon différentes modalités. La réponse ne peut être qu'anthropologique puisqu'elle touche à la symbolique de la prostitution des corps libres, à partir de la question 
reformulée ainsi : quelle fonction assurent les prostitué(e)s libres dans le système symbolique qui à Rome sert à représenter l'érotisme des corps libres, féminins et masculins? En effet se prostituer n'est pas une pratique comme une autre à Rome, elle marque d'infamie ces hommes et ces femmes nés libres, les privant du droit d'hériter aussi bien que de porter plainte pour viol ou insultes ${ }^{15}$. Elle crée ainsi une catégorie inférieure de citoyens.

Remarquons que la situation des prostitué(e)s libres est comparable à celle des acteurs qui eux aussi pouvaient à Rome aussi bien être libres qu'affranchis ou esclaves. D'une façon générale la question posée est celle de la concurrence dans une société hiérarchisée entre les hommes et les femmes libres mais infâmes d'une part et d'autre part les esclaves, au sein d'une même profession. Cette concurrence ne se maintient que si elle assure un rôle symbolique dans la configuration des marges de la société. La présence de citoyens libres et infâmes en bas de la hiérarchie sociale sert, entre autres, à définir les limites de la citoyenneté sans que se dessine une coupure franche ce qui permet de penser plus facilement le passage du dehors au dedans, des corps affranchis. Donc si des femmes et des hommes libres mais que leur profession rend infâmes, n'avaient pas joué un rôle de ce genre dans l'imaginaire de la cité romaine, il est clair que ces activités infamantes leur auraient été simplement interdites ce qui n'est le cas ni pour le théâtre ni pour la prostitution ni pour d'autres professions dégradantes qui posent le même problème. C'est pourquoi de même que nous avons vu au cours d'une étude précédente ${ }^{16}$ que l'acteur à Rome fonctionnait comme l'Autre de l'orateur et servait à définir et limiter la parole oratoire en la fondant en vérité par la masculinité libre du corps et en particulier de la bouche de l'orateur, on regardera dans cette brève étude de quelles figures honorables féminines ou masculines, les prostitué(e)s libres sont les Autres.

Ce qui semble d'emblée indiquer la position symbolique des prostituées libres dans l'espace public est le costume spécifique qui leur est imposé et qui sert à les distinguer des épouses honorables, les matrones, elles aussi revêtues d'un vêtement particulier.

La matrone seule a le droit de porter la stola, une longue robe qui lui tombe jusqu'aux pieds ${ }^{17}$ et d'avoir les cheveux attachés par des bandelettes ${ }^{18}$. La prostituée n'a pas droit à la stola et doit porter en public la toge sombre des hommes du peuple ${ }^{19}$. Cette différence vestimentaire est essentielle car elle protège le pudor - l'honneur - de la matrona de toute offense dans la rue. En effet quiconque attenterait par le geste ou la parole à l'honneur corporel de la matrone est susceptible d'être poursuivi en droit. En revanche si une femme libre et honorable n'affiche pas les signes de son statut elle ne peut revendiquer les droits qui y affèrent. La différence vestimentaire signale ainsi des différences de droit et de statut dans la cité, imposant des comportements différents à leur égard. Les unes méritent le respect, les autres ne peuvent se protéger ni de l'offense ni de la violence. Le corps des matrones est intouchable, voire sacré, et comme celui des citoyens protégés par la loi, celui des femmes prostituées même libres et ingénues comme celui des hommes libres et ingénus prostitués, acteurs ou gladiateurs, ne l'est pas.

21 L'opposition principale qui situe la meretrix dans la société romaine est donc celle qui la pose comme l'autre de la matrona. Cette opposition est visible de tous dans l'espace public. La toge portée par les prostituées n'a de valeur que lorsqu'elles sont libres, car pour les autres prostituées la question ne se pose pas. Affranchies ou esclaves, elles n'auraient de toute façon pas droit à la stola et aux privilèges qu'elle implique. 

un de ces objets sexuels achetés pour le plaisir des maîtres puis rejetés dès qu'ils ont passé l'âge imberbe et dont l'ignominie est la pire qui puisse se penser à Rome. Car si la prostituée est physiquement ignoble, du moins elle n'est pas liée socialement à ses clients. C'est pourquoi la liaison d'Antoine avec Curion est pire que son prétendu passé de garçon de trottoir. Esclave volontaire à cause de ce plaisir ignoble pour les Romains qu'est la soumissions sexuelle, il est entièrement assujetti à la volonté d'un autre, Curion, le comble du vice et de la faute morale pour un Romain. Totalement efféminé, Antoine sexuellement soumis à Curion est l'inverse d'une matrone qui par rapport à lui est donc dans une position masculine, la masculinité étant à Rome affaire de moralité et non de sexe. Remarquons que la soumission sexuelle volontaire que notre époque pourrait appeler « amour » est à Rome moralement pire que la prostitution, car elle ne peut se penser que comme un asservissement.

Dans l'imaginaire romain, la meretrix ingénue est donc l'autre de la matron $a^{22}$. Mais cette altérité n'est pas radicale elle va de paire avec une proximité potentielle. Ce que démontre l'histoire édifiante d'Hispala.

La matrone et la putain : histoire de Faecenia Hispala

Clio. Femmes, Genre, Histoire, 17 | 2003 
27 Rares sont chez les historiens anciens les passages même brefs concernant la prostitution. En fait les prostitué(e)s n'apparaissent pratiquement pas dans les textes qui se présentent non comme des fictions mais comme rapportant des événements réels, sauf parfois dans les discours d'accusation ${ }^{23}$.

28 L'histoire d'Hispala Faecenia que nous rencontrons largement développée chez Tite Live est donc un document unique, instructif autant par ce qui y est raconté que par son existence même. Une analyse détaillée du texte de son implicite social et culturel va nous permettre de voir comment une prostituée libre fonctionne comme l'autre de la matrona honesta, c'est-à-dire de l'épouse-mère dont le rang tient à son honneur et sa chasteté - pudor. En même temps ce texte présente le cas du passage, exceptionnel, d'un statut à l'autre puisqu'Hispala ayant commencé dans la vie comme jeune esclave prostituée finit en noble matrone, mariée et respectée de toute la cité. La figure d'Hispala donc bien que n'appartenant pas à la classe des prostituées ingénues rend compte de la raison de leur existence.

L'itinéraire d'une meretrix

Hispala est présentée d'emblée dans le récit par la formule «bien connue comme prostituée » - nobile scortum -, avant de devenir l'humble héroïne du fameux scandale des Bacchanales en 186 av. J.-C., tel que le raconte Tite Live ${ }^{24}$.

Elle commence sa vie comme esclave prostituée par son maître Faecenius. Sa situation est alors banale : achetée pour ses qualités physiques elle est louée par son propriétaire à des clients qui le payent, pour un banquet, une liaison de quelques jours ou de quelques mois. Elle ressemble alors trait pour trait aux courtisanes de comédie qui appartiennent à un marchand de filles comme celles du Pseudolus de Plaute par exemple. Le comportement de ces filles est toujours le même : assoiffées de plaisirs et ne songeant qu'à satisfaire leur maître elles soutirent tout l'argent qu'elles peuvent à leurs amants. Créatures sensuelles et cyniques, elles sont la propriété d'un ignoble marchand de filles, le leno. Elles appartiennent en toute légitimité culturelle au monde du plaisir et de l'infamie.

31 C'est à cette époque de sa vie qu'Hispala, très logiquement, fréquente avec sa maîtresse les banquets nocturnes des initiés à Bacchus. Ces fêtes que le texte de Tite Live dénonce comme des orgies où les femmes et les jeunes gens perdent leur honneur, n'ont rien de honteux pour une esclave, qui plus est, prostituée, dont l'honneur est perdu depuis toujours aussi bien par son statut servile que la profession imposée par son maître.

Ensuite, elle est affranchie mais conserve le même métier. Elle est alors semblable à un autre type de courtisanes de la comédie romaine: celles qui travaillent pour ellesmêmes, comme les sœurs Bacchis dans les Bacchides de Plaute. Tout aussi séduisantes et redoutables que leurs collègues esclaves, ces affranchies galantes ruinent leurs amants à leur profit, en les attirant dans des banquets dont ils font tous les frais. Une fois ruinés, elles les chassent. Ce qui est culturellement logique. En effet les prostituées et leurs amants sont dans de purs rapports marchands. Ils ne sont pas liés par des relations de don et de contre-don qui seules leur imposeraient une forme de loyauté amoureuse : fides et amor. C'est pourquoi dans la comédie romaine les garçons qui ont une amie régulière, cherchent à racheter la fille afin de se l'attacher en l'affranchissant. C'est la seule façon pour eux d'éviter la ruine car sinon il leur faudra sans cesse payer le marchand de filles chaque fois qu'ils désireront la voir. En la rachetant une fois pour toutes ils n'ont plus à payer, et ils l'affranchissent, car sinon elle deviendrait la 
propriété de leur père. Ainsi de clients dépendants ils deviennent le patron auquel leur maîtresse est toujours redevable, dans une relation de don.

En revanche une prostituée affranchie, si elle se doit à son patronus, reste dans des rapports marchands avec ses clients. Elle ne peut nouer avec eux des relations d'amour et de fidélité. C'est pourquoi les poètes romains se plaignent sans fin de l'infidélité de leurs maîtresses quand il s'agit de prostituées affranchies. L'exemple le plus connu est celui d'un amant de Cithaeris, Gallus, auquel Virgile destine une Bucolique plaintive ${ }^{25}$. Les chants désespérés qui ornent l'histoire littéraire sont bien souvent de simples artefacts culturels.

Or c'est ici qu'Hispala va se démarquer de ses perfides congénères et entrer dans l'histoire. Elle a une liaison avec un jeune homme qui habite non loin de chez elle, sur l'Aventin, Publius Aebutius, situation conforme aux habitudes romaines. Mais phénomène exceptionnel au lieu de soutirer de l'argent à son amant, elle l'entretient, car le pauvre garçon est orphelin de père et sa mère remariée à un certain Titus Sempronius ne donne pas de quoi vivre convenablement à son fils. En outre le beaupère a dilapidé l'héritage du père et cherche à se débarrasser d'Aebutius de peur d'avoir à rendre des comptes à sa majorité.

La conduite de la meretrix est célébrée par l'historien : son désintéressement la rend " digne » dit-il « de ne pas exercer ce métier » - non digna quaestu -, qu'on pourrait développer en «digne de ne pas vivre dans la solitude et l'ignominie de rapports marchands ». Ainsi Hispala inverse l'image habituelle de la meretrix affranchie, et déplace les rapports sociaux attendus. Elle se situe à l'égard d'Aebutius en position de donatrice, de bienfaitrice ${ }^{26}$ ou encore de "patronus»: don sexuel, don d'argent, protection contre une famille qui veut se débarrasser de lui.

Hispala est donc une courtisane paradoxale qui a créé du lien social entre elle et Aebutius alors qu'il n'appartenait pas à la gens de son ancien patron, comme si elle était née libre. Ce qui explique qu'elle fera preuve d'un comportement civique. En effet convoquée par le consul Postumius elle acceptera de dénoncer les crimes commis lors des Bacchanales et de témoigner contre les membres de la secte, ayant été autrefois initiée et ayant participé aux horribles fêtes.

Dans l'intervalle Hispala a vu son statut évoluer une troisième fois. Faecenius, son patron étant mort, sans doute sans enfants, elle s'est retrouvée libre de toute tutelle - in nullius manu - (9) et donc en droit de disposer comme elle veut de ses biens .qu'elle lègue en totalité à Aebutius. Autre paradoxe, car en général les satiristes dénoncent les hommes libres qui donnent tous leurs biens à une courtisane au point que le droit interdit les legs en leurs directions ${ }^{27}$. Elle se substitue donc à la mère d'Aebutius, particulièrement déficiente, et même à son père mort. C'est pourquoi quand elle apprend que le garçon va être initié aux Bacchanales sur les conseils de sa mère et de son beau-père qui cherchent ainsi à se débarrasser de lui, en le privant de son honneur - pudicitia - et peut-être même de la vie - uita - (10), elle prend peur et lui dit tout ce qu'elle sait sur les orgies nocturnes et les crimes des Bacchants. Et c'est ainsi que grâce à elle, la secte pourra être anéantie. De bienfaitrice d'un citoyen dont elle sauve la masculinité, elle devient bienfaitrice de la cité tout entière.

Une bienfaitrice de la patrie

38 Hispala sera récompensée par la cité à la hauteur de ses services (19). Elle sera libérée de toutes les contraintes et de l'infamie attachées à son statut d'affranchie et d'ancienne prostituée. En particulier elle aura le droit de se marier en dehors de la gens 
de son patronus, elle pourra choisir son tuteur comme une veuve ingénue et son mari ne subira aucune infamie en fonction de son passé. Elle acquiert donc de fait la dignité de matrone. Comme elle est privée de gens et donc de défenseur en cas d'offense, c'est la Cité, par l'intermédiaire de ses magistrats supérieurs, qui va lui tenir lieu de gens. Le sénat décide, en effet, que les consuls et les préteurs en charge, puis leurs successeurs, veilleront à ce qu'aucun tort - iniuria - ne lui soit fait. Voilà donc Hispala entrée dans la sphère civique, ce qui va bien au-delà de ce qui est accordé aux matrones les plus nobles dont le rôle dans la cité est limité à certains rituels religieux ${ }^{28}$. Hispala est devenue en quelque sorte « fille de la patrie».

La figure d'Hispala, esclave prostituée, affranchie accédant à la dignité de matrone, même si elle est historique, était nécessaire à la logique du récit de Tite Live. Il fallait en effet pour qu'éclatât le scandale des Bacchanales, que quelqu'un ou plutôt quelqu'une assurât le passage entre d'une part le monde nocturne et parallèle des assemblées bacchiques, entièrement du côté du plaisir, de l'effémination, du stuprum et de l'impudicitia, du privé, de la cupidité marchande et de la prostitution, et d'autre part le monde diurne, masculin et civique, de la générosité du don, du clientélisme politique, des magistrats, du sénat, du public et de la loi. Le caractère paradoxal de cette "prostituée généreuse » lui permet d'appartenir à ces deux mondes. Son passé de prostituée servile l'a introduite dans la secte des Bacchants, où elle a été conduite par sa maîtresse et initiée (10 et 12). Ensuite, une fois affranchie, elle ne s'y est plus rendue ce qui prouve une moralité exceptionnelle. En empêchant ensuite Aebutius d'être initié, elle sauve son honneur d'homme et protège un citoyen. Ce qui manifeste qu'elle a changé d'espace et a intégré les réseaux de solidarité qui unissent les citoyens entre eux par les bienfaits. Elle quitte le monde du crime et de l'argent pour celui de la fidélité et de la reconnaissance. Enfin elle sauve la collectivité tout entière, grâce à son courage ; elle atteint ainsi à la vertu civique par excellence, la uirtus.

Remarquons que le trajet accompli par Hispala n'aurait pas pu être effectué par une impudica ingénue, qui aurait choisi volontairement de se soumettre sexuellement. Hispala malgré son statut et son infamie serviles, se révèle ne pas avoir l'âme d'une esclave ni la cupidité d'une meretrix. Elle ne cherche pas à exploiter financièrement son amant mais elle n'est pas non plus asservie sexuellement à Aebutius, en termes contemporains «elle ne l'aime pas » et c'est pourquoi elle peut le sauver comme le ferait un ami.

41 Le détail du récit rend compte du trajet extraordinaire accompli par Hispala dans la cité et des difficultés qu'il implique. En particulier, au moment où elle décide de sauver Aebutius, elle reste trop éloignée de la sphère civique, publique et masculine pour ne pas avoir besoin de médiations, quand elle doit entrer en contact avec le consul. Cela ne se fera que grâce à une chaîne féminine qui passe par la tante paternelle de Publius, Aebutia qui n'est pas noble, elle habite sur l'Aventin comme Hispala, mais est honorablement connue comme " une femme honnête, avec les mœurs d'autrefois » probam et antiqui moris feminam - (11). C'est ensuite Sulpicia, la belle-mère du consul Postumius, de noble famille et aux mœurs sévères - grauem et nobilem feminam - (12) qui sert de seconde intermédiaire. On voit ici à l'œuvre une sociabilité spécifique des femmes qui peut relier malgré la distance morale et sociale, la meretrix affranchie et la matrona patricienne.

Ce qui explique finalement comment est possible la promotion sociale d'Hispala. La moralité des femmes qui lui servent d'intermédiaires, est son sauf-conduit. La probitas, 
d'Aebutia et la grauitas, la nobiltas de Postumia sont des vertus constitutives de la uirtus, "masculines" c'est-à-dire civiques et non propres aux hommes. La uirtus et son contraire l'effémination constituent un axe sur lequel se déplace Hispala, aussi bien dans sa vie que pour rencontrer le consul et sauver la cité. Il est, en effet, remarquable que l'infamie sexuelle relève en dernier recours d'une décision politique, qu'elle ne soit pas l'effet d'une pollution "magique» mais d'un jugement éthique. Certes normalement l'infamie causée par la prostitution est irréversible, car le corps libre est sacré29 mais ce n'est pas une nécessité au-dessus de la loi des hommes. Quand il le faut la cité peut en décider autrement. Ce qui prouve bien qu'il s'agit d'une règle fonctionnelle interne à la culture sans dimension transcendante. L'histoire d'Hispala n'est que le renversement, exceptionnel mais possible, du passage de l'état de matrone à celui de prostituée, lui prévu par la loi. En effet, une matrone romaine peut, par simple déclaration devant les édiles, prétendre faire profession de prostituée pour perdre son statut de matrona honesta et se libérer de toute accusation d'adultère si elle a des amants et pour protéger ceux-ci de l'accusation de stuprum ${ }^{30}$.

Il est remarquable que de tels passages n'aient pas lieu dans la comédie romaine où l'on ne voit jamais un jeune homme épouser une meretrix ${ }^{31}$ ni une matrone devenir une prostituée. En effet la société comique est une utopie domestique et ignore ce qui est l'essentiel d'une société pour les Anciens, la dimension civique qui inclut à Rome non seulement l'activité politique et judiciaire. C'est pourquoi aucun personnage ne peut changer de statut et qu'on ne peut y voir une meretrix acquérir l'honorabilité d'une matrone. A la différence de la société comique, celle des historiens est une cité réduite à sa dimension. On ne s'étonnera donc pas de ne pas voir y figurer des prostituées sinon exceptionnellement, à l'occasion d'événements politiques extraordinaires mêlant le public et le prive, le politique et le domestique.

La belle et édifiante histoire d'Hispala montre comment d'une certaine façon la matrona et la meretrix ont partie liée mais seulement dans la sphère publique, dans la cité hiérarchisée.

Corps féminins, corps masculins : la louve et le soldat

Les Romains ne distinguent pas les prostitué(e)s selon leur sexe tant qu'il s'agit d'affranchi(e)s ou d'esclaves ${ }^{32}$. Le terme neutre de scortum $^{33}$ sert souvent à les désigner de telle sorte que dans certains récits il est impossible de savoir le sexe de la créature, car il n'importe pas.

Un exemple célèbre de cette indistinction sexuelle est l'histoire du proconsul Flaminus, et de son scortum ${ }^{34}$. Selon certains historiens il aurait emmené avec lui Philippe le punique, «scortum nobile » comme l'appelle Tite Live dont le nom montre qu'il s'agit d'un affranchi ou d'un étranger, ce qui revient au même. Au cours d'un banquet, le jeune homme qui est couché aux côtés de son amant, exige de lui par caprices de voir égorgé un Gaulois. Selon d'autres l'exécution a lieu pour complaire à une femme de mauvaise vie "famosam mulierem", elle aussi désignée comme "scortum». Certaines versions mêmes, comme le texte de Cicéron, ne précisent pas le sexe du scortum. Le crime du gouverneur Flaminus est le même qu'il soit commis pour complaire à sa maîtresse ou à son amant.

Qu'en est-il des prostitués ingénus? La prostitution masculine au sens strict exclut symboliquement, comme la prostitution féminine, les ingénus qui la pratiquent de tout réseau de solidarité, ce qui est évidemment plus grave pour un homme que pour une femme. Celui-ci ne peut pas se faire des amis ni créer des liens de reconnaissance 
réciproque, il est cantonné dans les rapports marchands. Sénèque prend l'exemple d'un prisonnier dont un prostitué a payé la rançon :

"Que doit faire un prisonnier lorsque le prix de sa rançon lui est promis par un homme au corps prostitué et dont la bouche est infâme? Accepterais-je d'être sauvé par un être souillé ? Ensuite, une fois sauvé, lui serai-je reconnaissant - gratial referam ? Vais-je associer à ma vie un être obscène ? Je refuserais d'associer à ma vie mon sauveur? Selon moi voici ce qu'il faut faire: j'accepterais d'un homme comme lui l'argent nécessaire à mon rachat mais je l'accepterais comme un prêt et non comme un don beneficium - ; je lui rembourserai ensuite la somme et si un jour il se trouve en danger, je le sauverai ; mais je n'irai pas jusqu'à le traiter en ami, car l'amitié unit des semblables, je ne le considérerai pas comme mon sauveur mais comme mon créancier à qui je sais que je dois rendre ce qu'il m'a donné35 ».

Le texte est clair : un prostitué, même ingénu, est l'autre du citoyen dans le cadre de la sociabilité des hommes libres. Il est exclu de tout rapport de don et de contre-don, ce qui le place dans les marges de la société au-delà même de l'affranchi qui a des relations d'officium avec son patron. Il est un individu libre mais solitaire, réduit aux relations commerciales avec les autres hommes, comme le marchand de filles ou le soldat de la comédie romaine, autres exclus de l'imaginaire romain.

Economiquement, le prostitué masculin est donc semblable à la prostituée féminine. Mais il se différencie d'elle sur les points suivants: il ne porte pas de vêtement spécifique, il ne fait pas de déclaration officielle pour être inscrit comme prostitué et il n'y a pas de terme particulier pour le désigner qui serait le pendant du mot meretrix. Nous développerons ce dernier point car il nous permet de voir le prostitué comme l'autre du soldat.

51 Le terme meretrix appartient originellement à la langue du droit même si ses emplois sont plus larges. Le mot sert à désigner la profession des prostituées ingénues qui se déclarent au préteur, et forment une classe recensée publiquement. Le terme est un dérivé en -trix, féminin des dérivés en -tor qui servent à former à partir de racines verbales des substantifs désignant des agents sociaux, comme praetor, le préteur, ou editor, qui préside les jeux, ou encore sutor, le cordonnier. Pourquoi n'existe-t-il pas de meretor ${ }^{36}$ ?

La réponse tient à un autre usage du verbe dans un contexte militaire car le verbe mereor ne peut servir à désigner la prostitution masculine ingénue parce le terme est déjà utilisé pour dire "toucher sa solde ». Comme la toge prend un sens opposé selon qu'elle est portée par un citoyen ou une ingénue prostituée, on retrouve avec le verbe mereor une concurrence entre les signes valorisants de la masculinité et les signes infamants de la prostitution féminine.

L'armée réunit des hommes sans toge, sans maison et sans épouse. Les matronae sont exclues de l'espace militaire et donc la masculinité civique des soldats s'oppose directement à l'effémination des prostitué(e)s ${ }^{37}$. On voit ainsi le corps du jeune soldat protégé en droit dans les camps de la séduction de ses supérieurs avec autant de soin que le corps des matrones dans les rues de la Ville. C'est ce que montrent les récits des historiens relatant les procès intentés à des officiers par des jeunes recrues victimes de stuprum $^{38}$. Si l'accusé peut prouver que sa victime est connue pour se prostituer, il sera normalement acquitté, sinon son séducteur sera gravement condamné : 
54 «(Caius Cornelius, un centurion primipile) comme il avait eu des relations sexuelles stupri commercium - avec un très jeune ingénu - ingenuo adulescentulo - fut arrêté et enchaîné. Il en appela aux tribuns et dit qu'il reconnaissait avoir eu des relations sexuelles - stupro - mais que si on le libérait sous caution il prouverait que le garçon avait l'habitude de se prostituer ouvertement - adulescens ille palam atque aperte corpore quaesitum factisset ${ }^{39}$ ".

Inversement un autre jeune homme qui accuse un magistrat de stuprum obtient sa condamnation grâce à son maintien pudicus, prouvant ainsi son honneur physique masculin - pudor - et donc la réalité du crime. Un tribun de la plèbe Caius Scantinus avait incité le fils de l'édile curule, Marcus Claudius Marcellus à avoir des relations sexuelles avec lui - stupro - :

56 «Cité comme accusé, Scantinius fut condamné sur le seul témoignage de celui qu'il avait tenté de corrompre. Le jeune homme - iuuenem - monté sur la tribune garda les yeux baissés vers la terre sans rien dire et il est sûr que ce silence pudique - uerecundo silentio - fit beaucoup pour qu'il obtînt la condamnation de son adversaire ${ }^{40} »$.

57 Le soldat et le/la prostitué(e) reforment dans l'espace des camps, le couple symbolique opposant la masculinité vertueuse et l'effémination voluptueuse, omniprésent dans tous les espaces de la civilisation romaine et constitutif de la culture. Ce couple est figuré par le loup et la louve. Le loup, animal de Mars, symbolise la dimension sauvage de la sociabilité des jeunes hommes dans la guerre ${ }^{41}$. Au cours de la fête des Lupercales, des jeunes gens sont travestis en sauvages nus, en hommes-loups, incarnant rituellement une humanité primitive, sans femme et sans maison, une société uniquement masculine et guerrière, sous l'égide de Mars. Un épisode du mythe de Romulus et Rémus est associé à ce rituel : les jeunes Luperques reproduiraient les deux jumeaux, guerriers et bergers nomades, avant la fondation de la Ville, élevés sur un Palatin encore sauvage par une prostituée, lupa, compagne d'un berger, après avoir été allaités bébés par une véritable lupa, descendue de sa grotte creusée au flanc de la colline, le Lupercal.

58 En latin la lupa, la louve, désigne couramment la prostituée dont la maison est aussi appelée un lupanar. Les prostituées prennent souvent un surnom qui rappelle le loup : que ce soit Lycoris aimée de Gallus ou Messaline qui se fait appeler Lycisca ${ }^{42}$.

On voit ainsi comment symboliquement la lupa, la femelle du lupus, est son inverse : le loup figure la vertu militaire, le corps sacré du jeune soldat, la louve incarne le corps prostitué de l'un ou l'autre sexe. Que la louve soit devenue l'animal emblématique de Rome montre comment est centrale la figure de la prostitution (féminine ou masculine indifféremment) à Rome qui structure l'espace féminin et urbain, en s'opposant à la matrone, et l'espace masculin de la guerre en s'opposant au soldat ${ }^{43}$. 


\section{BIBLIOGRAPHIE}

DÉTIENNE Marcel, SVENBRO Jesper, 1979, « Les loups au festin ou la cité impossible », Marcel Détienne et Jean-Pierre Vernant (éd.), La Cuisine du sacrifice en pays grec, Gallimard.

DUPONT Florence, 1989, La Vie quotidienne du citoyen romain, Hachette.

DUPONT Florence, 2000, L'Orateur sans visage, PUF.

DUPONT Florence, 2002, « Le lait du père », dans Philippe Moreau (éd.), Corps romains, Grenoble.

EDWARDS Catharine, 1993, The Politics of immorality in Ancient Rome, Cambridge University Press.

GARDNER Jane F., 1986, Women in Roman Law and Society, Indiana University Press.

GOUREVITCH Danielle, 2001, Les Femmes dans la Rome antique, Hachette.

MYEROWITZ Molly, 1992, "The domination of Desire. Ovid's parua tabella and the Theatre of Love », in Richlin Amy (ed.), Pornography and Representation in Greece and Rome, Oxford University Press, pp. 131-158.

PAILLER J. M., 1988, Bacchanalia. La répression de 186 av. J.-C. à Rome et en Italie, EFR.

RICHLIN Amy ed., 1992, Pornography and Representation in Greece and Rome, Oxford University Press.

ROBERT Jean-Noël, 1986, Les Plaisirs à Rome, Belles Lettres.

ROBERT Jean-Noël, 1997, Eros romain, Belles Lettres.

SALLES Catherine, 1982, Les Bas-fonds de l'antiquité, Robert Laffont.

SCHEID John, 1991, « Indispensables étrangères », Michelle Perrot, Georges Duby dir., Histoire des femmes en Occident, tome I, L'Antiquité, Plon, pp. 405-438.

VANOYÉKÉ Violaine, 1990, La Prostitution en Grèce et à Rome, Les Belles Lettres.

\section{NOTES}

1.En français nous ne citerons que pour mémoire les livres de Catherine Salles, 1982 ; Jean-Noël Robert, 1986 et 1997 ; Danielle Gourévitch, 2001. Même le livre dirigé par Amy Richlin (1992) est décevant sur Rome, en particulier l'article de Molly Myerowitz, qui prétend découvrir, derrière le discours officiel et les représentations moralisatrices, une réalité érotique que l'archéologie dévoilerait (p. 138 et suiv.). Non seulement victime de l'illusion de l'intime, elle croît aussi à une « évolution des mœurs » au sein du couple matrimonial sur le modèle historique du XXe siècle occidental.

2.Pour citer le terme utilisé par Paul Veyne, à tort selon nous à cause de son anachronisme ethnocentrique.

3.Sénèque, Les Bienfaits, VI, 32,1.

4.Juvénal, Satires VI, 115-132.

5.Cicéron, Pro Caelio : Clodia n'est appelée meretrix que par métaphore morale. Elle a une vie meretrico more $(38,17)$. Palam sese in meretricia uita conlocarit $(49,2)$ c'est-à-dire qu'elle fréquente des hommes avec lesquels elle n'a aucun lien familial : uirorum 
alienissorum. Quintilien (Institution oratoire, XI, 4) commente cette appellation de meretrix appliquée par Cicéron à Clodia comme un exemple d'amplification oratoire.

6.Tacite, Historiae, 1, 13, 15.

7.Plinius l'Ancien, Naturalis Historia, IX, 119, 6.

8.Catharine Edwards (1993) n'aborde la dimension économique de la prostitution que du point de vue de la morale somptuaire.

9.Sénèque le rhéteur Controverses 4, Préface 10 : Impudicitia in ingenuo crimen est, in seruo necessitas, in liberto officium.

10.Digeste XXV, 7, 1 .

11.Catharine Edwards, 1993 : 188-191.

12.Sur l'otium, Florence Dupont, 1989 : 203 et suiv.

13. Cicéron, De senectute, L, 14. : epularum aut ludorum aut scortorum uoluptates.

14. Columelle, De re rustica I, 8, 2 : otiis, campo, circo, theatris, aleae, popinae, lupanaribus.

15. Catharine Edwards cite par exemple la lex Iulia municipalis CIL, I, 593, ligne 123 qui interdit de magistrature « queiue corpore quaestum fecit » (Edwards $1993: 71$ et 123-126). Cf. aussi Violaine Vanoyéké, 1990.

16.Florence Dupont, 2000.

17.Par exemple, Cicéron, Philippiques, II, 18, 44.

18.Servius Ad Aen. VII, 403.

19.Schol. Horace, Satires, I, 2, 63 (par exemple).

20.La toge de l'orateur s'oppose aussi aux vêtements bariolés de la prostituée pour figurer la rudesse du discours viril, fût-il mal dégrossi, à l'effémination d'un discours trop orné : Tacite, Dialogue des orateurs, XXVI, 1.

21.Cicéron, Philippiques, 2, 44,-45 : Numpsisti uirilem quam statim muliebrem togam reddidisti. Primo volgare scortum; certa flagiti merces nec ea parva; sed cito Curio intervenit qui te a meretricio quaestu abduxit et, tamquam stolam dedisset, in matrimonio stabili et certo conlocavit. Nemo umquam puer emptus libidinis causa tam fuit in domini potestate quam tu in Curionis.

22.L'opposition entre matrona et meretrix fournit à la rhétorique de multiples figures antithétiques récurrentes dans toute sorte de discours. Ainsi les larmes de la matrone et les rires de la prostituée : Pline L'Ancien, Histoire Naturelle, 34, 71, 1, ou l'alliance des prostituées et des matrones campaniennes pour aider les Romains, Valère Maxime, $\mathrm{V}$, 2, 1 ou encore cette formule de Cicéron, De finibus, II, 12, 2: «tamquam meretricem in matronarum coetum, sic uolutptatem in virtututm adducere " (introduire un plaisir dans une réunion de vertus c'est faire entrer une prostituée dans une assemblée de matrones).

23.Par exemple chez Cicéron (discours contre Verrès, Antoine ou Catilina). Mais en revanche elles sont sureprésentées dans les textes de fiction comme la comédie romaine ou les controverses oratoires. Ce qui mesure leur poids dans l'imaginaire romain et indique que la présence des prostitués est essentiellement à penser dans leur dimension symbolique.

24.Sur l'affaire des Bacchanales, Cf. J. M. Pailler, 1988.

25.Née vers 70 av J.C., Lycoris est d'abord esclave. Affranchie sous le nom de Volumnia Cythaeris par son patron (affranchi lui-même), Publius Volumnius Eutrapelus. Lycoris, autrement dit « la louve grecque » devient Cythaeris, la Cithéréenne, c'est-à-dire « d'Aphrodite ». Elle fut aimée de Gallus, premier préfet d'Egypte, puis de Brutus et Marc Antoine qu'elle suivit en Gaule. Cf. Servius Ad Virg Buc. 10, 1. Marc Antoine la présente comme sa femme Volumnia, ce qui scandalise Cicéron. Chantée par les poètes 
Virgile, Ovide. Et surtout Gallus (4 livres d'élégies). Cf. Martial VIII, 73, 6 et Virgile, Bucoliques, 10.

26.Sur la notion romaine de beneficium comme équivalent du don et du contre-don, cf. Sénèque, De Beneficiis.

27.Jane F. Gardner, 1986 : 132-134.

28.John Scheid, 1991.

29.Le corps d'un citoyen doit non seulement être à l'abri du stuprum mais encore de tout châtiment corporel qui laisserait des traces infamantes comme les coups de fouet sur son dos : Valère Maxime VI, $1,9$.

30.Tacite, Annales II, 85 et Suétone, Tibère, 35.

31.Il arrive seulement qu'un personnage de prostituée se révèle être en fait une jeune fille pure, ce qui se voit à son masque qui a deux profils, par exemple dans le Charançon de Plaute.

32.Un esclave peut aussi être voué à la prostitution par son maître comme Hispala, puis être affranchi. C'est le cas par exemple de Publius Atilius Philiscus « in pueritia corpore quaestum a domino facere coactum » (Valère Maxime, VI, 1, 6).

33.Sur le sens et l'emploi de scortum: Varron, De lingua latina, 7, 84, Prophyrion, ad Horat. carm. II, 10, 21.

34.Tite Live, XXXIX, 42-43 ; Sénèque le Rhéteur, Controverses, IX, 2, Cicéron De senectute, XII, 42, Valère Maxime, II,9,3 ; Plutarque Cato maior, 17, 1-8 et Flamininus, 18, 3-6.

35.Sénèque, De beneficiis, II, 21, 1-2

36.En revanche l'adjectif meritorius/a sert à désigner un ou une prostitué. Cf. Cicéron, Philippiques, II, 105, 7, « Ingenui pueri cum meritoriis, scorta inter matres familiae uersabantur ».

37.Sur la présence des prostitué(e)s dans les camps, cf. Valère Maxime, II, 7, 1.

38. Valère Maxime, VI, 1 et Quintilien, Declamationes Maiores, 3.

39.Valère Maxime, $\mathrm{VI}, 1,10$.

40.Valère Maxime, VI, $1,7$.

41.Marcel Detienne et Jesper Svenbro, $1979: 215$ et suiv.

42.Juvénal Satires VI, 122.

43.Que la louve romaine soit aussi représentée en nourrice - ce qu'elle n'est pas primitivement - renvoie au statut très particulier des nourrices à Rome (Dupont, 2002).

\section{RÉSUMÉS}

Le problème historique majeur posé par l'étude de la prostitution à Rome est celui de l'existence de prostitué(e)s libres dans une société esclavagiste. Puisque des corps serviles, ou affranchis, masculins et féminins étaient disponibles en grand nombre, aussi bien dans les demeures des hommes libres que dans les maisons de prostitution, comment se fait-il que des femmes nées libres aient renoncé à leurs privilèges et statut de matrones? Comment se fait-il aussi que la société ait institutionnalisé ce renoncement en prévoyant d'enregistrer officiellement ces prostituées d'origine libre? L'explication proposée ici est que les prostitué(e)s libres assument une fonction symbolique qui est d'être l'autre du soldat comme l'acteur est l'autre de l'orateur. 
Ces «parias » de la société romaine, toujours "efféminés » servent à marquer les marges de l'humanité civilisée dans la continuité, à définir les hommes, libres et adultes et donc «masculins» par ce qu'ils ne sont pas mais ce qu'ils pourraient par malheur, devenir des « effeminés » ou leur équivalent, des prostituées libres.

The main historical problem that occurs when studying prostitution in Rome is the existence of free prostitutes in a slave State. Since servile bodies as well as male and female freed slaves were available in large numbers, in the homes of free men and in brothels, why did born-free women give up their privilege and their status of wives of Roman citizens? How could society institutionalise this renouncement by officially registering these prostitutes of free origin? The explanation given here is that free prostitutes took on a symbolic function which was to be the Other of the soldier just like the actor is the Other of the speaker. Always "feminine", these society outcasts were used to define the margins of civilised mankind in its continuity. They were used to define the free and adult men, who were thus " masculine" through what they were not, but what they might unfortunately have become, "feminine" or their equivalent, free prostitutes.

\section{AUTEUR}

\section{FLORENCE DUPONT}

Florence DUPONT est professeur de littérature et civilisation romaines à l'URF Sciences des Textes et Documents de l'Université de Paris 7, directeur de programme au Collège International de Philosophie, chercheur au Centre Louis Gernet EHESS-CNRS UMR 8567. 